\title{
Heart and Lungs in COPD. Close friends in real life - separate in daily medical practice?
}

\author{
M. Lusuardi, G. Garuti, M. Massobrio, L. Spagnolatti, S. Bendinelli
}

ABSTRACT: Heart and Lungs in COPD. Close friends in real life - separate in daily medical practice? M. Lusuardi, G. Garuti, M. Massobrio, L. Spagnolatti, S. Bendinelli.

Chronic obstructive pulmonary disease (COPD) is associated with a 2-3 times higher rate of cardiovascular disorders (CVD) which is independent of other risk factors. A low $\mathrm{FEV}_{1}$ is a specific predictor of mortality as a result of cardiac causes, even stronger than increased cholesterol: for each $10 \%$ reduction of $\mathrm{FEV}_{1}$, cardiovascular mortality increases by $28 \%$. The main causes of death among COPD patients are of cardiovascular origin. COPD and CVD have two major risk factors in common - advanced age and tobacco smoking. The search for a pathogenetic link between the two conditions focuses mainly on systemic extension of pulmonary inflammation.
Despite such a frequent association, pulmonologists and cardiologists in both the clinical and the research settings often underestimate the importance of a correct diagnosis and severity stratification of the two combined conditions. Spirometry, in particular, is largely underprescribed. Missed diagnosis and severity stratification, incomplete knowledge of adverse drug events and lack of resources lead to undertreatment of patients combining COPD and CVD, and in particular, the underuse of beta-blockers, inhaled bronchodilators and rehabilitation. Clinical studies focusing on this group of patients should be promoted in the future to test therapies and manage options. Furthermore, efforts must be made to improve the present standards of care, which falls short of recommended levels, starting from the often-neglected use of spirometry to confirm a diagnosis of COPD.

Monaldi Arch Chest Dis 2008; 69: 1, 11-17.

Key words: Chronic obstructive pulmonary disease, Cardiovascular disorders, Spirometry, Bronchodilators, Beta-blockers.

Unit of Cardiology and Pulmonary Rehabilitation, AUSL Reggio Emilia, S. Sebastiano Hospital, Correggio, Italy.

Correspondence: M. Lusuardi, S. Sebastiano Hospital, 42015 Correggio (RE), Italy; e-mail: lusuardimi@ausl.re.it

The anatomical and functional interdependence of the heart and lungs is one of the basic notions in medical studies; acute cardiogenic pulmonary edema or pulmonary embolism or "cardiac asthma" are clinical conditions which emphasise the heart-lung interaction. Quite often, however, organ specialists fail to bear this in mind in their daily routine practice, particularly when dealing with chronic patients.

Chronic obstructive pulmonary disease (COPD) and cardiovascular disorders (CVD) are among the most widespread medical conditions, from an epidemiological point of view: in the USA in 2003, 71.3 million Americans had some form of cardiovascular disease (about $25 \%$ of residents) and about 1 million deaths were due to a CVD, mainly coronary artery disorders (53\%) and stroke (17\%) [1]. According to Mannino DM et al., the prevalence of COPD in the adult U.S. population is $14 \%$, based on a spirometric inclusion criterion of $\mathrm{FEV}_{1} / \mathrm{FVC}<0.7$ [2]. COPD and CVD could easily co-exist in the same individual simply as a matter of probabilistic chance; however, it is well known that lung and heart diseases have a close reciprocal influence. Nevertheless, pulmonologists and cardiologists often focus on their own field of specialisation when treating patients, concentrating on the disease rather than on the patient. This may be due to hyperspecialistic post-graduate education (at least in Italy), lack of time and resources, inadequate organization of diagnostic and management procedures, and/ or criteria for scientific research that tends to focus on a single variable at a time, eliminating all interfering factors. In the scientific literature, only a limited number of papers have considered in some way the association of COPD and CVD: a gross search in Medline [3] found 31,560 citations for the term COPD, $1,424,172$ citations for the term "cardiovascular disorders" and 3,817 references for the combination of the two terms. Despite an increasing level of interest, literature on "cardiovascular disorders and COPD" in the latest 12 months (197 papers) has not increased in proportion to articles on COPD $(2,231)$ or cardiovascular disorders $(47,072)$. In addition approximately one out of every four published articles is a review or an editorial article (this paper making no exception). In addition, many studies on treatments exclude the main comorbidities as interfering variables. It is therefore 
very difficult to find published evidence-based data on how to treat chronic patients with different disorders in real life. The intention of the present article is not to review the pathophysiology of lung-heart interaction but to focus the attention of clinicians on the discrepancies between the extent of the problem and the present inadequate standards of care.

\section{A Pulmonologist's point of view}

In Italy, COPD affects about $4.5 \%$ of the general population [4]. Cardiovascular complications (i.e. secondary disorders directly dependent on the basic disease from a pathogenetic point of view) of COPD are well known, in particular pulmonary hypertension and cor pulmonale due to chronic hypoxia. Right heart failure is a strong risk factor for death in COPD [5]. The only effective treatment at present of cor pulmonale due to obstructive lung disorders is long-term oxygen therapy, an option Cardiologists generally associate with Respiratory Medicine and thus infrequently prescribe. In a recent Italian paper on a cohort of moderate to severe COPD patients admitted to Pulmonary departments for an exacerbation of disease, $51 \%$ of subjects had chronic respiratory failure and were therefore at risk of cor pulmonale; all were discharged on long-term oxygen therapy [6]. Among comorbidities, 37\% had hypertension and $22 \%$ coronary heart disease. The average number of comorbidities was 1.1 per patient [6].

Other CVD such as coronary artery disease, hypertension, stroke, and left heart failure should be considered as co-morbidities (a medical condition existing simultaneously with but independently of another condition in patients) [7] unless a direct and strong etiopathogenetic link is found with COPD. According to different patient series, approximately $10 \%$ to $40 \%$ of COPD patients have signs of left ventricular dysfunction and a large percentage of COPD patients die of a cardiovascular cause. Review data from the literature demonstrate that the main causes of death in mild to moderate COPD patients are lung cancer and cardiovascular disease, while moderate to very severe COPD patients $\left(\mathrm{FEV}_{1}<60 \%\right)$ die primarily of respiratory failure [5].

Cardiovascular causes are also a major cause of hospitalisation of COPD patients: according to the Lung Health Study, $42 \%$ of first and $44 \%$ of second hospitalisations were due to CVD in patients with mild-moderate COPD, respiratory causes accounting for only $14 \%$ of admissions [8].

It has been repeatedly demonstrated that poor lung function per se, independent of age, gender and smoking, is associated with an increased cardiovascular risk [5]. A meta-analysis including $>80,000$ patients revealed that subjects with the lowest lung function quintile have almost doubled the risk of cardiovascular mortality (RR 1.77) in comparison with patients in the highest lung function quintile [9].

\section{How do Pulmonologists consider the treatment} of COPD patients with concomitant CVD?

Severe patients with chronic respiratory failure and right heart failure receive, in general, longterm oxygen therapy as recommended, at least in Italy. With regard to other standard treatments, there are data in the literature reporting large discrepancies between an ideal approach as set forth in the guidelines and current management of COPD: overprescription of inhaled corticosteroids, underprescription of long-acting bronchodilators, minimal consideration of education/ self-management and pulmonary rehabilitation in treatment plans [10]. Specific investigations on COPD patients with CVD are very limited. In a cohort of 1,529 patients with left ventricular dysfunction, inhaled short-acting $\beta 2$-agonists put patients at risk of increased mortality or heart failure exacerbations [11]. A recent meta-analysis concluded that $\beta 2$-agonists in asthma and COPD increase the risk of adverse cardiovascular events, such as precipitation of ischemia, congestive heart failure, arrhythmias and sudden death, one of the mechanisms being related mainly to an increase in heart rate and hypokaliemia [12].

With regard to cardiologic treatments, $\beta$-blockers are frequently underprescribed due to the concern about inducing bronchoconstriction. It is true that this can occur in asthmatic subjects or COPD patients with large airflow variability, but a recent meta-analysis demonstrated that cardioselective $\beta$-blockers can be safe in COPD patients, with no or very limited long-term effects on pulmonary function [13].

\section{A Cardiologist's point of view}

In the IN-CHF (Italian Network - Chronic Heart Failure) registry, the prevalence of COPD (clinical diagnosis) among 8,627 patients followed up for 12 months was $13.3 \%$. Compared with CHF patients without COPD, respiratory patients had a statistically significant worse trend in different outcomes (total mortality, $p=0.007$, cardiovascular mortality $p=0.012$, total hospitalizations $p=$ 0.0019 , total + in-hospital mortality $p=0.0001)$. One possible explanation is the undertreatment of CHF patients with COPD with $\beta$-blockers (9.2 versus $20.1 \%$ ), despite the current availability of cardioselective drugs (such as atenolol, metoprolol, bisoprolol and acebutolol), which have minimal impact on respiratory function [14].

According to the international literature, the prevalence of COPD in patients with CHF varies from $10 \%$ to $32 \%$, and in patients with a previous diagnosis of COPD, CHF is often overlooked as a cause of dyspnea and echocardiography is not performed [15]. In fact, the risk ratio of developing $\mathrm{CHF}$ in COPD patients is 4.5 as compared with age-matched non-COPD controls, after adjustments for cardiovascular risk factors [15].

Interestingly, COPD patients have an increased risk of cardiovascular morbidity and mortality that is not influenced by common risk factors 
such as tobacco smoking. A reduction in $\mathrm{FEV}_{1}$ (forced expiratory volume in $1 \mathrm{sec}$.) is as strong a predictor of cardiovascular mortality as a specific marker like increased serum cholesterol [15]. For each $10 \%$ reduction in $\mathrm{FEV}_{1}$ cardiovascular mortality increases by $28 \%$ [8].

In a large cohort of 4,284 patients admitted to hospital for coronary heart disease, mortality rates at 3-year follow up were far higher in cases of a concomitant diagnosis of COPD (21\%) than in subjects without COPD (9\%) [16]. These data together with several other studies in the literature contribute to defining COPD as a powerful independent risk factor for cardiovascular morbidity and mortality [17].

Cardiologists are particularly wary of $\beta 2$-agonists, given their potential to cause arrhythmias and to precipitate pump failure or ischemia, with a risk of sudden death [12]. The same is true for theophylline, which, given its narrow therapeutic range, is at present considered a second option to an inhaled bronchodilator [18]. On the other hand, bronchodilation may have positive effects on cardiovascular function by alleviating dynamic hyperinflation, which reduces intrathoracic pressure, thus improving venous return and cardiac output. Reduction of lung hyperinflation also unloads the ventilatory muscles and lessens the effort of breathing, in particular during exercise. A safe alternative to $\beta 2$ agonists is represented by anticholinergics. The only long-acting anticholinergic available at the moment, tiotropium, has been shown to improve cardiac and pulmonary function during exercise in COPD [19]. Safety data of tiotropium in the long term has not demonstrated significant cardiac adverse events [20]. Further studies are needed to evaluate bronchodilator therapies in populations with concomitant COPD and CVD.

With reference to the use of $\beta$-blockers, there is evidence that when cardiologic patients have a diagnostic label of COPD they are often denied the prognostic benefit of these agents. In a recent study by Egred $\mathrm{M}$ et al. on subjects with acute coronary syndrome, $46 \%$ of patients did not receive $\beta$-blockers, $33 \%$ of them because of a COPD label. Interestingly, however, only $62 \%$ had their diagnosis confirmed with pulmonary function tests or reviewed by a chest physician. COPD patients receiving $\beta$-blockers represented only $16 \%$ of all subjects with COPD [21].

It is basic evidence that medications currently associated with cardiovascular risk reduction (statins, angiotensin-converting enzyme (ACE) inhibitors or angiotensin receptor blockers (ARBs)) show the potential to attenuate different forms of lung injury. With this as their starting point, Mancini et al. in a recent retrospective study used large databases to evaluate whether patients with concomitant COPD could also benefit from these drugs. The combination of statins and either ACE inhibitors or ARBs, in particular, reduced both CVD and pulmonary outcomes, specifically COPD hospitalisations and total mortality, thus prompting the need to confirm these findings in randomised clinical trials [22].
Is there a common etiopathogenetic link?

COPD and CVD share the two greatest risk factors in modern western culture: advanced age and tobacco smoking. There is also evidence that environmental pollution may have important respiratory and cardiovascular effects. Acute exposure to high levels of particulate air pollution induces an oxidative stress at the pulmonary level, which causes systemic inflammation and disrupts the hemostatic-fibrinolitic balance. Soon after exposure, the risk of acute cardiovascular events increases and lasts for days. Similarly, chronic exposure to air pollution is associated with a long-term risk of adverse cardiovascular outcomes [23].

The main mechanism by which smoking acts in non-neoplastic disorders is mediated by inflammation. Smoking is able to induce systemic activation of the inflammatory cascade but only genetically susceptible subjects are considered to develop disease. After smoking cessation, inflammation may improve, but in many cases inflammation becomes self-perpetuating [24].

COPD includes inflammation in its definition [18]. In addition, atherosclerosis is now considered to have an important chronic inflammatory component $[25,26]$. C-reactive protein (CRP), a common marker of systemic inflammation now considered a major risk indicator for cardiovascular disease, has been found to be higher even in stable COPD and could contribute to the progression of atherosclerosis [17]. There is also data regarding the possible role of acute lung inflammation in inducing acute cardiac events by acting on the coagulation cascade through an elevation of plasma fibrinogen and serum IL-6 levels (such as in COPD exacerbations) [27] or by acting on the atheromatous plaques through production of proinflammatory acutephase proteins and stimulation of the bone marrow (such as in subjects exposed to air pollutants) [28]. Recent studies have found some evidence of a role of a lung pathogen, Chlamydia Pneumoniae, in atherosclerosis, but the clinical relevance of these data in risk stratification, prevention, and (antibiotic) therapy of atherosclerosis is still doubtful [29]. Not only smoking or COPD or lung injury in general are associated with cardiac adverse events; there are other disorders inducing systemic inflammation that have no risk factors in common (such as rheumatoid arthritis or other collagen vascular disease in non-smokers).Therefore, inflammation is probably the common final pathway of different conditions associated with CVD [5].

Despite potential links based on common risk factors and derived biological data, mortality trends would seem to speak against a correlation between COPD and CVD: age-adjusted death rates in the USA in the period 1965-1998 saw a decrease of $59 \%$ for coronary heart disease, $64 \%$ for stroke, and $35 \%$ for other CVD, as opposed to an increase of COPD mortality of $163 \%$ in the same period. In the period 1990-2020, CVD mortality is expected to remain stable at 1 st (ischemic heart disease) and 2nd (cerebrovascular disease) place, while COPD mortality will rise from $6^{\text {th }}$ to $3^{\text {rd }}$ 
place [30]. Obviously, not only common etiopathogenetic factors may influence these reciprocal trends but also social and medical aspects, as well as criteria for administrative registration of the causes of death. Nevertheless, such a large increase in COPD mortality should probably have a direct relationship with CVD mortality if the two conditions are indeed closely linked.

If a close "inflammatory" link between CVD and COPD does exist, we can expect substantial benefits from treatments targeting inflammation. Different cardiovascular drugs originally acting on non-inflammatory mechanisms have shown benefits to both CVD and COPD, such as in the abovementioned case of statins, with a probable effect on the inflammation in the atheromatous plaque and in the lungs [22].

Among the reference anti-inflammatory drugs, inhaled corticosteroids are recommended in moderate to severe cases of COPD $\left(\mathrm{FEV}_{1}<\right.$ $50 \%$ ) with repeated exacerbations [18], but such a therapy is largely overprescribed outside guidelines indications [10]. A re-analysis of pooled data from different papers demonstrated that inhaled corticosteroids reduce all cause mortality [18]. A recent paper highlighted a remarkable reduction in cardiovascular deaths in COPD subjects receiving inhaled steroids or a combination of inhaled steroids and bronchodilators as compared to bronchodilators alone, but the analysis was performed on retrospective data [31]. A rigorous prospective study has been published recently in which no significant effect of inhaled corticosteroids was found on cardiovascular mortality in COPD, at the same time confirming a positive effect on COPD exacerbations and health status [32]. These data do not seem to confirm systemic inflammation as a link between COPD and CVD, but we are all aware that the problem is probably related to our limited knowledge of the different inflammation phenotypes. A significant example is represented by the different patterns of inflammation in the bronchial mucosa of patients with asthma (mucosal infiltration by eosinophils and CD4 lymphocyte, good response to corticosteroids) and COPD (prevalence of mononuclear cells and CD8 lymphocytes in the bronchial mucosa, neutrophils in the lumen, bad responsiveness to corticosteroids) [18].

\section{How to improve standards of care for patients with coexisting COPD and CVD?}

\section{Diagnostic standards}

An elderly patient, current or former smoker, seeking medical advice for progressing exercise dyspnea is a common presentation in hospitals and medical offices. It is theoretically correct to proceed with a differential diagnosis between COPD and heart failure and a severity stratification but how consistently are a co-existence and reciprocal interference of the two conditions taken into account? In many cases, it is not so much a question of whether it is COPD or heart failure, but to what extent COPD and heart failure impact the global health status assessment.

This is not a naïve question, because treatment strategies require an accurate evaluation of risk and benefits of the different therapies and each intervention must be proportionate to the severity of each condition in the context of a global evaluation of co-morbidities [33].

COPD is often misdiagnosed, mainly because spirometry is underutilised, even by pulmonologists themselves [34]. In the face of persisting dyspnea despite optimised cardiological treatment, Cardiologists infrequently refer patients to a pulmonary function laboratory for a spirometry. For their part, Pulmonologists are mindful of right heart failure, but frequently neglect left heart disorders and underuse $\beta$-blockers.

An interesting retrospective study by $M$. Damarla et al. demonstrated that standards in the diagnosis of COPD or CHF at a Centre in Boston differed widely: only $30 \%$ of the patients with a first diagnosis of COPD had had a spirometry performed within the previous 7 years compared with $78 \%$ of patients with a first diagnosis of $\mathrm{CHF}$ who had had a 2D-echocardiography performed in the same period. Among patients with both diagnosis of COPD and CHF, $48 \%$ underwent 2D-echocardiography only, $2 \%$ spirometry only, $34 \%$ had both tests, while $16 \%$ underwent neither test [35].

Although the best diagnostic strategy has yet to be identified, standards will improve only if the knowledge of epidemiological data, common risk factors and respective treatment option force clinicians to consider COPD in any CVD patient and vice versa. From a practical point of view, a detailed case history and physical examination may help considerably, despite the fact that several findings can be present in both COPD and left ventricular dysfunction and that the two conditions may reciprocally influence the performance and interpretation of instrumental evaluations [33]. Prescription of 2D-echocardiography and spirometry should nevertheless be optimised.

A cardiopulmonary exercise test is an important tool in the global functional evaluation of the patient in order to stratify morbidity and mortality risks (e.g. in the decisional process for long-term medical treatment or thoracic surgery and transplantation) and to plan rehabilitation programmes. According to recent evidence, levels and changes in partial pressures of mixed-expired $\mathrm{CO}_{2}$, endtidal $\mathrm{CO}_{2}$, and their ratios during cardiopulmonary exercise testing are distinctive of COPD, left ventricular failure, and pulmonary vascular diseases and are explained by the differing pathophysiologies of V/Q mismatching in these disorders [36]. As a second option, where facilities for cardiopulmonary exercise test are not available, exercise field tests (6-minute walk test and shuttle test) are an appropriate, simple tool to evaluate disability and obtain outcome measures for rehabilitation programmes [38].

In our integrated unit of Cardiology and Pulmonology we have been able to develop over time 
Table 1. - Inadequate standards of care in the management of COPD patients in general and in patients with both COPD and $\operatorname{CVD}\left(^{*}\right)$

\begin{tabular}{l|l}
\hline Underutilised items & Overutilised items \\
\hline Spirometry & $\begin{array}{l}\text { Short-acting bronchodilators } \\
\text { 2-D echocardiography }\left(^{*}\right)\end{array}$ \\
$\begin{array}{l}\text { Inhaled corticosteroids alone or in combination } \\
\text { with long-acting } \beta 2 \text {-agonists }\end{array}$ \\
$\begin{array}{l}\text { Education - self management } \\
\text { Smoking cessation }\end{array}$ \\
$\begin{array}{l}\text { Long-acting bronchodilators } \\
\beta \text {-blockers }(*)\end{array}$ \\
$\begin{array}{l}\text { Pulmonary rehabilitation } \\
\text { Palliative care in the terminally ill }\end{array}$ \\
\hline
\end{tabular}

a more consistent diagnostic approach to cardiorespiratory patients. Our working protocols, for example, require that all smokers with CVD undergo spirometry with bronchodilator test, that all COPD patients with borderline respiratory failure undergo 2D echocardiography to exclude pulmonary hypertension, and that all patients with moderate to severe left heart failure are studied for nocturnal sleep disturbances. Furthermore, close follow up of severe left heart failure may include evaluation of lung diffusing capacity as an index of early decompensation. A further benefit is that we feel more at ease when prescribing pharmacologic treatments (e.g. bronchodilators in CVD patients or $\beta$-blockers in COPD patients, or oxygen in severe heart failure) and non pharmacologic therapies, such as rehabilitation and mechanical ventilation, for severe patients with both COPD and heart failure.

\section{Treatment standards}

Removal of risk factors, and specifically tobacco smoking, is the first common task of cardiologists and pulmonologists: smoking cessation is the most cost-effective intervention for both COPD and CVD but it is often neglected in clinical practice $[18,38]$. A strong positive message to smokers can be represented by the rapid decline in the risk of acute cardiovascular events after quitting smoking.

Prescribing $\beta$-blockers to COPD patients or bronchodilators to CVD patients should never be excluded a priori, as often happens now, but a correct diagnosis and accurate cost-benefit analysis in the clinical setting are mandatory. Patients who have never experienced wheezing episodes are much more likely to tolerate and benefit from cardioselective $\beta$-blockers. Inhaled bronchodilators are recommended in symptomatic COPD at any stage; long-acting bronchodilators are more effective and convenient than short-acting agents in regular treatments [18]. Whenever a risk of cardiac adverse events is present, anticholinergic agents should be preferred due to their safety profile [12, $20]$. The combination of bronchodilators with different mechanisms and duration of action may increase the degree of bronchodilation as compared to the individual classes of agents and reduce the risk of side effects [18]. Therefore, for patients already on treatment with $\beta 2$-agonists who may need an increase in their bronchodilator therapy to control dyspnea, the addition of an anticholinergic may be a preferable option.

Oxygen is recommended in cases of chronic respiratory failure with persistent hypoxaemia at rest $\left(\mathrm{PaO}_{2}<55 \mathrm{mmHg}\right.$ or $<60 \mathrm{mmHg}$ in presence of cor pulmonale or policytemia), but patients with COPD and heart failure may often experience isolated nocturnal hypoxaemia. Research is needed to evaluate whether nocturnal oxygen therapy may be of benefit to these patients (e.g. in terms of mortality reduction, improvement of disability and quality of life).

Pulmonary rehabilitation should be prescribed to all symptomatic COPD patients willing to participate [18]; cardiac rehabilitation plays an important role in chronic or post-acute heart disorders but only a minority of COPD or CVD patients have access to rehabilitation programmes [6, 38]. Respiratory and cardiac rehabilitation share several features, in particular exercise training as the central component and improvement of health status as the main outcome.

The most evident deficiencies in the standards of care for COPD patients are summarised in table 1 .

In conclusion, although several guidelines on COPD have been produced in the last few years, the problem of concomitant COPD and CVD has been only marginally addressed. As the two conditions are frequently associated, this has had important consequences on the management of patients. Although the etiopathogenetic mechanisms that might link COPD and CVD are still poorly understood, the question of their association is critical to daily clinical practice. Therefore, clinical studies should be promoted by pulmonologists, cardiologists and general practitioners 
working together to test diagnostic and management protocols specifically focusing on this group of patients. Secondly, committed effort and resources are needed to improve the present standards of care, which fall short of those recommended, starting with the very basic use of spirometry in the diagnosis of COPD.

Acknowledgments. The Authors are grateful to the non-profit organisation "Amici del Cuore" of Correggio (RE), Italy, for their invaluable support in the continuous upgrading of the instrumentation facilities of the Cardiopulmonary Unit of S. Sebastiano Hospital, Correggio, Italy.

\section{References}

1. Thom T. Heart Disease and Stroke Statistics-2006 Update: A Report From the American Heart Association Statistics Committee and Stroke Statistics Subcommittee. Circulation 2006; 113: e85-e151.

2. Mannino DM, Gagnon RC, Petty TL, Lydick E. Obstructive lung disease and low lung function in adults in the United States: data from the National Health and Nutrition Examination Survey, 1988-1994. Arch Intern Med 2000; 160: 1683-9

3. PubMed online, accessed October 31, 2007.

4. ISTAT. Indagine multiscopo sulle famiglie. Roma, Istituto Nazionale di Statistica, 1994.

5. Sin DD, Anthonisen NR, Soriano JB, Agusti AG. Mortality in COPD: role of comorbidities. Eur Respir $J$ 2006; 28: 1245-1257.

6. Lucioni C, Donner CF, De Benedetto F, et al. I costi della broncopneumopatia cronica ostruttiva in Italia: la fase prospettica dello studio ICE. Pharmacoeconomics Italian Research Articles 2005; 7: 119-134.

7. http://en.wikipedia.org/wiki/Comorbidity.

8. Anthonisen NR, Connett JE, Enright PL, Manfreda J. Lung Health Study Research Group. Hospitalization and mortality in the Lung Health Study. Am J Respir Crit Care Med 2002; 166: 333-339.

9. Sin DD, Wu L, Man SF. The relationship between reduced lung function and cardiovascular mortality: a population-based study and a systematic review of the literature. Chest 2005; 127: 1952-1959.

10. O'Donnell DE, Hernandez P, Aaron S, et al. Canadian Thoracic Society COPD Guidelines: Summary of highlights for family doctors. Can Respir J 2003; 10: 183185 .

11. Au DH, Udris EM, Fan VS, Curtis JR, McDonell MB, Fihn SD. Risk of mortality and heart failure exacerbations associated with inhaled $\beta$-adrenoceptor agonists among patients with known left ventricular systolic dysfunction. Chest 2003; 123: 1964-1969.

12. Salpeter SR, Ormiston TM, Salpeter EE. Cardiovascular effects of $B$-agonists in patients with asthma and COPD. A Meta-Analysis. Chest 2004; 125: 2309-2321.

13. Salpeter SR, Ormiston TM, Salpeter EE. Cardioselective beta-blockers in patients with reactive airway disease: a meta-analysis. Ann Intern Med 2002; 137: 715725.

14. Pulignano G, Del Sindaco D, Tavazzi L, et al. Clinical features and outcomes of elderly outpatients with heart failure followed up in hospital cardiology units: Data from a large nationwide cardiology database (IN-CHF Registry). American Heart Journal 2002; 143: 45-55.

15. Le Jemtel TH, Padeletti M, Jelic S. Diagnostic and therapeutic challenges in patients with coexistent chronic obstructive pulmonary disease and chronic heart failure. J Am Coll Cardiol 2007; 49: 171-180.
16. Berger JS, Sanborn TA, Sherman W, Brown DL. Effect of chronic obstructive pulmonary disease on survival of patients with coronary heart disease having percutaneous coronary intervention. Am J Cardiol 2004; 94: 649-651.

17. Sin DD, Man SFP. Chronic obstructive pulmonary disease as a risk factor for cardiovascular morbidity and mortality. Proc Am Thorac Soc 2005; 2: 8-11.

18. Rabe K, Hurd S, Anzueto A, et al. Global strategy for the diagnosis, management, and prevention of chronic obstructive pulmonary disease. GOLD Executive Summary. Am J Respir Crit Care Med 2007; 176: 532-555.

19. Travers J, Laveneziana P, Webb KA, Kesten S, O'Donnell DE. Effect of tiotropium bromide on the cardiovascular response to exercise. Respir Med 2007; 101: 2017-2.

20. Casaburi R, Mahler DA, Jones PW, et al. A long-term evaluation of once-daily inhaled tiotropium in chronic obstructive pulmonary disease. Eur Respir J 2002; 19: 217-224.

21. Egred M, Shaw S, Mohammad B, Waitt P, Rodrigues E. Under-use of beta-blockers in patients with ischemic heart disease and concomitant chronic obstructive pulmonary disease. $Q$ J Med 2005; 98: 493-497.

22. Mancini GBJ, Etminan M, Zhang B, Levesque LE, FitzGerald JM, Brophy JM. Reduction of morbidity and mortality by statins, angiotensin-converting enzyme inhibitors, and angiotensin receptor blockers in patients with Chronic Obstructive Pulmonary Disease. $J$ Am Coll Cardiol 2006; 47: 2554-2560.

23. Mittleman MA. Air pollution, exercise, and cardiovascular risk. N Engl J Med 2007; 357: 1147-1149.

24. Yanbaeva DG, Dentener MA, Creutzberg EC, Wesseling G, Wouters EFM. Systemic Effects of Smoking. Chest 2007; 131: 1557-1566.

25. Ross R. Atherosclerosis - an inflammatory disease. $N$ Engl J Med 1999; 340: 115-126.

26. Willerson JT, Ridker PM. Inflammation as a cardiovascular risk factor. Circulation 2004; 109: II2-II10.

27. Wedzicha JA, Seemungal TA, MacCallum PK, et al. Acute exacerbations of chronic obstructive pulmonary disease are accompanied by elevations of plasma fibrinogen and serum IL-6 levels. Thromb Haemost 2000; 84: 210-215.

28. van Eeden SF, Tan WC, Suwa T, et al. Cytokines involved in the systemic inflammatory response induced by exposure to particulate matter air pollutants $\left(\mathrm{PM}_{10}\right)$. Am J Respir Crit Care Med 2001; 164: 826-830.

29. Franz-Josef Neumann F-J. Chlamydia pneumoniae-atherosclerosis link a sound concept in search for clinical relevance. Circulation 2002; 106: 2414

30. Murray CJ, Lopez AD. Alternative projections of mortality and disability by cause 1990-2020: Global Burden of Disease Study. Lancet 1997; 349: 1498-504.

31. Macie C, Wooldrage K, Manfreda J, Anthonisen NR. Inhaled corticosteroids and mortality in COPD. Chest 2006; 130: 640-646.

32. Calverley PMA, Anderson JA, Celli B, et al. Salmeterol and Fluticasone Propionate and Survival in Chronic Obstructive Pulmonary Disease. N Engl J Med 2007; 356: 775-789.

33. Rutten FH, Cramer M-JM, Lammers J-WJ, Grobbee DE, Hoes AW. Heart failure and chronic obstructive pulmonary disease: an ignored combination? Eur J Heart Fail 2006; 8: 706-711.

34. Lusuardi M, Orlandini D. Underutilization of spirometry for the diagnosis of COPD. Chest 2008 133: 313-4.

35. Damarla M, Pinto-Plata VM, Celli BR. COPD and spirometry: CHF and 2-D Echo; the great disconnect. Chest 2004; 126: 919S.

36. Hansen JE, Ulubay G, Chow BF, Sun XG, Wasserman $\mathrm{K}$. Mixed-expired and end-tidal $\mathrm{CO}_{2}$ distinguish between ventilation and perfusion defects during exercise 
testing in patients with lung and heart diseases. Chest 2007; 132: 977-83.

37. American Thoracic Society. Guidelines for the SixMinute Walk Test. Am J Respir Crit Care Med 2002; 166: 11-117.

38. Balady GJ, Williams MA, Ades PA, Bittner V, Comoss P, Foody JM, Franklin B, Sanderson B, Southard D; American Heart Association Exercise, Cardiac Rehabilitation, and Prevention Committee, the Council on Clin- ical Cardiology; American Heart Association Council on Cardiovascular Nursing; American Heart Association Council on Epidemiology and Prevention; American Heart Association Council on Nutrition, Physical Activity, and Metabolism; American Association of Cardiovascular and Pulmonary Rehabilitation. Core components of cardiac rehabilitation/secondary prevention programs: 2007 update. Circulation 2007; 115: 2675-2682.

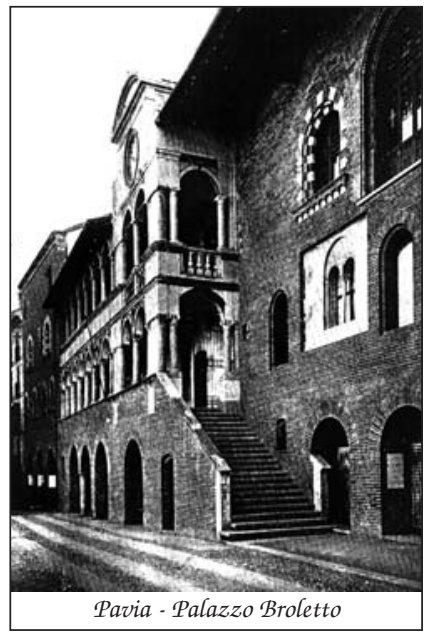

